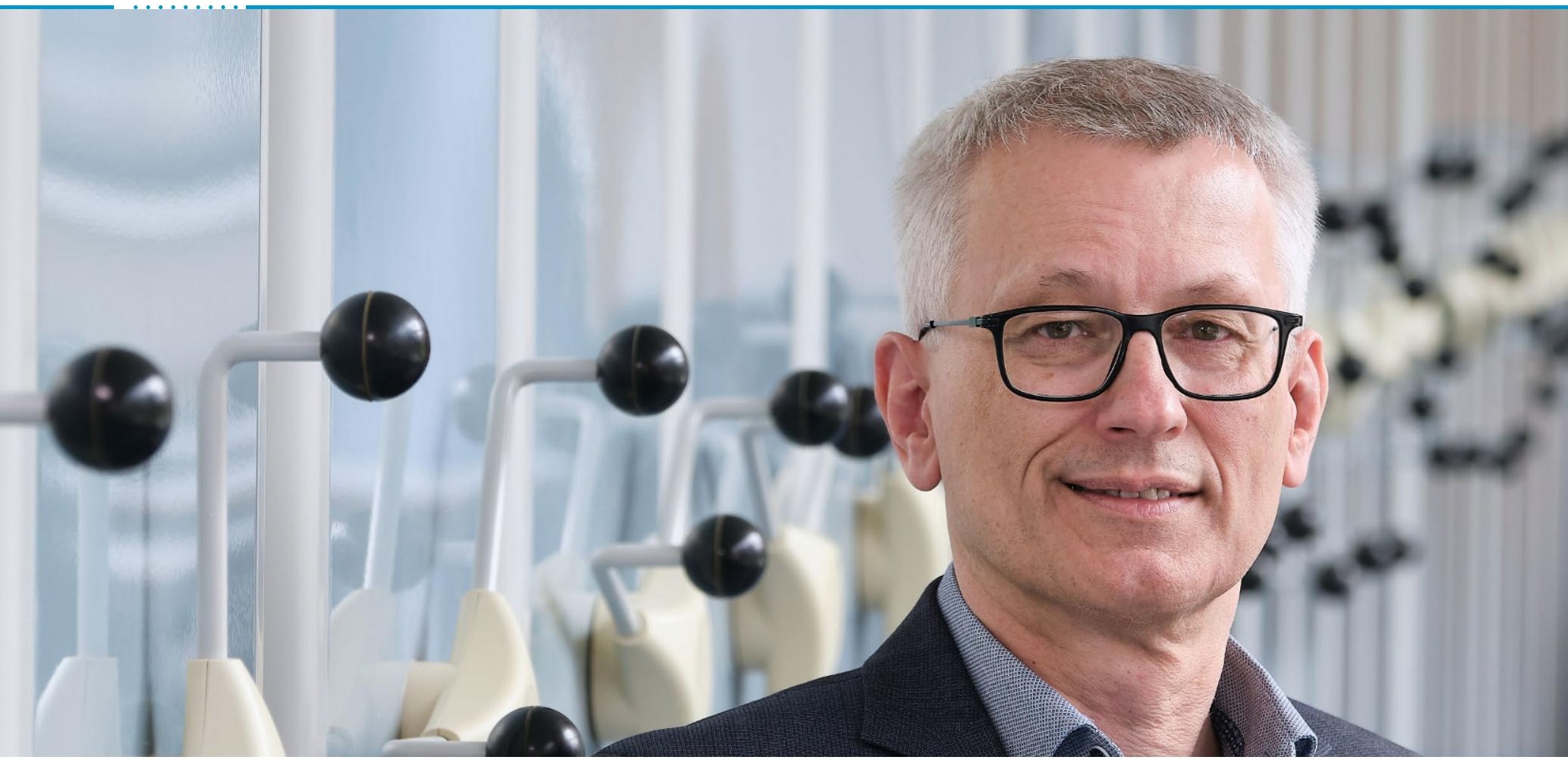

\title{
Sicher therapieren trotz komplexer Medikation
}

\author{
Interview mit Prof. Dr. Ulrich Jaehde, Leiter der Abteilung \\ Klinische Pharmazie am Pharmazeutischen Institut \\ der Rheinischen Friedrich-Wilhelms-Universität Bonn
}

Die Sicherheit der Therapie vor allem mit onkologischen Medikamenten liegt Prof. Dr. Ulrich Jaehde, Bonn, sehr am Herzen. Deshalb forscht er mit seinen Kollegen intensiv an Modellen, wie Krebspatienten unnötige unerwünschte Wirkungen und Arzneimittelinteraktionen erspart bleiben können. Seine Ziele sind ein strukturiertes, für jeden Patienten maßgeschneidertes Medikationsmanagement und eine gut abgestimmte interprofessionelle Zusammenarbeit.

best practice onkologie: Wie ist es dazu gekommen, dass Sie sich der onkologischen Forschung zugewandt haben?

U. Jaehde: Nach meinem Studium der Pharmazie und der Promotion an der Freien Universität Berlin ging ich zunächst als Postdoc an die Universität Leiden und beschäftigte mich bis dahin vor allen Dingen mit Antibiotika. Zurück in Berlin hatte ich danach die Möglichkeit, im Bereich der Klinischen Pharmazie eine eigene Arbeitsgruppe aufzubauen. Mein damaliger Chef hat mir völlig freie Hand gelassen, mein Arbeitsgebiet selbst festzulegen. Ich wollte mich thematisch erweitern und hinzukam, dass kurz zuvor meine Mutter mit 52 Jahren an Brustkrebs gestorben war. Das hat mich sehr geprägt. Daraufhin nahm ich mir vor, das Thema Krebs als Forschungsgebiet zu wählen. Denn ich hatte diese Ohnmacht gespürt, dass von medizinischer und pharmazeutischer Seite nur wenig für meine Mutter getan werden konnte. Das war und ist immer noch eine Motivation für mich, auf dem Gebiet zu arbeiten.

best practice onkologie: Wie hat sich das Nebenwirkungsprofil im Zeitalter von zielgerichteter und Immuntherapie verändert? U. Jaehde: Zunächst einmal muss man sagen, dass Chemotherapie ja auch heute noch verwendet wird. Das Spektrum der medikamentösen Therapie ist jedoch durch zielgerichtete und Immuntherapien erheblich erweitert worden. Heute werden alle diese Therapien in Abhängigkeit von der Tumorerkrankung nebeneinander oder auch in Kombination eingesetzt. Damit ist die medikamentöse Tumortherapie spezifischer und insgesamt wirksamer geworden. Das bedeutet aber auch, dass wir es nicht mehr nur mit den klassischen Nebenwirkungen der Chemotherapie wie etwa Knochenmarksuppression und Erbrechen zu tun haben, sondern dass durch die neueren Substanzgruppen ganz andere $\mathrm{Ne}$ benwirkungen hinzugekommen sind. Bei der zielgerichteten Therapie sind das beispielsweise charakteristische Hauterscheinungen und bei der Immuntherapie mit Checkpointinhibitoren verschiedene Autoimmunreaktionen. Das Nebenwirkungsmanagement ist somit durch die neuen Therapien und die Vielzahl 
der möglichen Kombinationen noch anspruchsvoller und komplexer geworden.

\section{》) Das Nebenwirkungs- management ist durch die neuen Therapien noch anspruchsvoller und komplexer geworden}

best practice onkologie: In Bezug auf Nebenwirkungen tritt immer mehr die Erfassung von PROs („patient-reported outcomes") in den Vordergrund. Wie lässt sich dieses Instrument weiter ausbauen?

U. Jaehde: Nebenwirkungen werden in der Regel vom Arzt anhand der „Common-Terminology-Criteria-for-Adverse-Events(CTCAE)-Kriterien“ des $\mathrm{Na}$ tional Cancer Institute (NCI) eingeschätzt. Im letzten Jahrzehnt ist man zunehmend dazu übergegangen, auch die Patienten $\mathrm{zu}$ fragen, wie es ihnen geht. Für diesen Zweck hat das NCI eine PRO-Version der CTCAE-Kriterien entwickelt. Die PROs werden immer wichtiger, in klinischen Studien werden diese heute meistens miterhoben. Wir haben hier in Zusammenarbeit mit dem NCI eine Validierungsstudie mit einem deutschsprachigen Kern-ItemSet aus dem PRO-CTCAE-Fragenkatalog durchgeführt [1]. Insgesamt sind es 124 Items zu 78 Symptomen, mit denen man die Patienten natürlich nicht alle behelligen kann. Das ist auch gar nicht nötig, weil nicht jeder Patient die gleiche Wahrscheinlichkeit hat, bestimmte Nebenwirkungen zu bekommen. Deshalb arbeiten wir an spezifischen Item-Sets für Patienten mit bestimmten Tumorentitäten. Wir haben uns bereits mit dem multiplen Myelom sowie dem Mamma- und Prostatakarzinom beschäftigt. Zurzeit spezifizieren wir den PRO-CTCAE-Fragebogen für das Kolorektalkarzinom. Dafür ermitteln wir anhand einer Befragung, welche Items bei welchen Patienten besonders relevant sind. Damit können wir die Anzahl der Fragen deutlich reduzieren, beispielsweise auf eine Anzahl von 20 bis 30 .

best practice onkologie: Können die PROs auch in der täglichen Praxis genutzt werden?

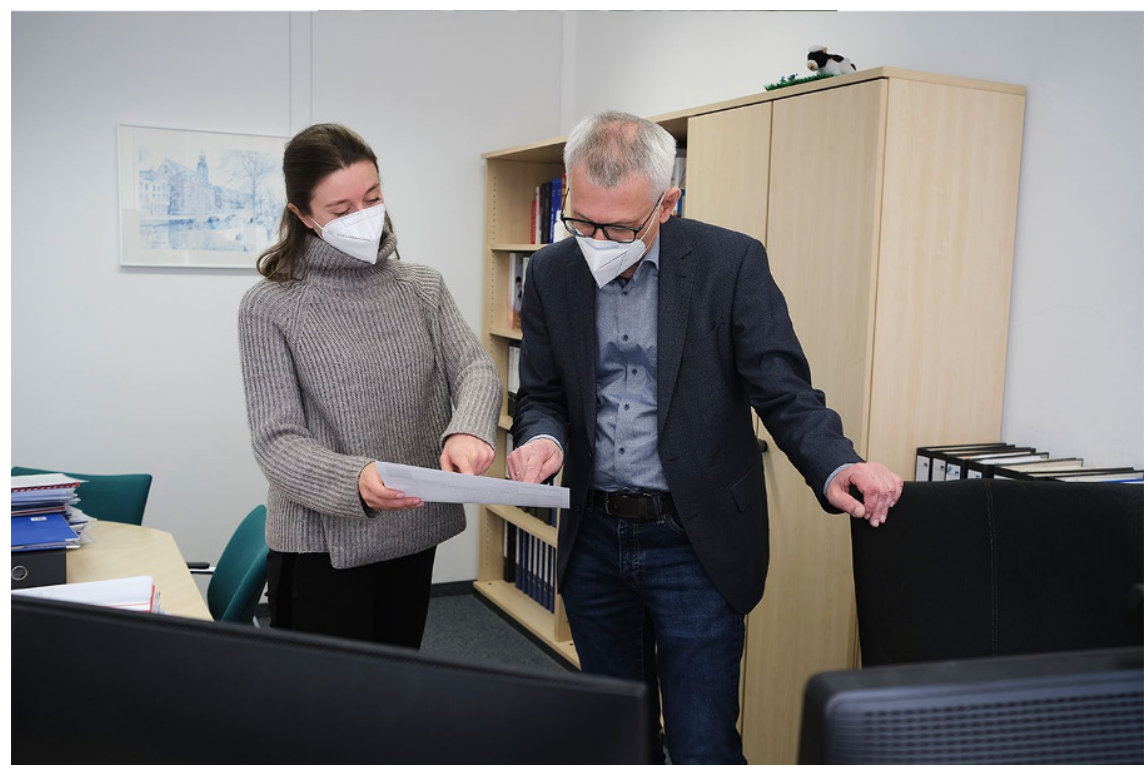

Anhand eines Medikationsplans werden Sicherheitslücken aufgedeckt

U. Jaehde: Überwiegend werden die PROs in Studien benutzt. Es ist aber durchaus sinnvoll, sie auch im Praxisalltag in das Symptom- und Nebenwirkungsmanagement aufzunehmen, das ist auch ein Thema, an dem wir momentan arbeiten. Die Patienten können über bestimmte Apps angeben, welche Symptome sie haben und wie stark diese sind. Diese Angaben werden dann an den behandelnden Arzt übermittelt, der daraufhin entscheiden kann, ob er eingreifen muss, ob er also beispielsweise die Dosis einer Substanz reduzieren oder sogar die Therapie wechseln muss. Vor Kurzem wurde eine randomisierte Studie aus Großbritannien publiziert, die gezeigt hat, dass eine wöchentliche elektronische Symptomerhebung das körperliche Wohlbefinden der Patienten verbessert [2].

\section{》) Es ist sinnvoll, PROs im Praxisalltag in das Symptom- und Neben- wirkungsmanagement aufzunehmen}

best practice onkologie: Sie entwickeln mit Ihrer Arbeitsgruppe pharmakometrische Modelle. Wie lässt sich damit die Sicherheit und Wirksamkeit der medikamentösen Tumortherapie verbessern?
U. Jaehde: Pharmakometrische Modelle beschreiben zeitliche Verläufe, bekannt sind vor allem pharmakokinetische Modelle, mit denen PlasmakonzentrationsZeit-Kurven beschrieben werden. Mit solchen Modellen lässt sich dann vorhersagen, wie sich die Plasmakonzentration wahrscheinlich verändert, wenn die Dosis erhöht oder reduziert wird oder sich die Nieren- oder Leberfunktion verändert. Durch Einbeziehung pharmakodynamischer Größen, wie z. B. Biomarkerkonzentrationen oder Blutdruckwerte, können diese Modelle erweitert werden. Das hat den Vorteil, dass man nicht nur Plasmakonzentrationen, sondern auch Wirkungen vorhersagen kann. Wir beschäftigen uns jetzt mit der nächsten Generation von Modellen. Damit wollen wir noch eine Ebene weiter gehen von pharmakodynamischen Parametern zu klinischen Effekten wie dem Ansprechen auf eine Tumortherapie oder Nebenwirkungen. Die Erwartung ist, dass wir damit Vorhersagen $\mathrm{zu}$ Wirksamkeit und Verträglichkeit eines Medikaments für bestimmte Patientenpopulationen oder sogar einzelne Patienten machen können. Mit diesen Modellen lassen sich auch Studien besser planen.

best practice onkologie: Wozu kann man solche Modelle im Praxisalltag anwenden?

U. Jaehde: Im Praxisalltag kann man diese Modelle im Rahmen eines sogenannten Therapeutischen Drug Monitorings 
(TDM) nutzen. Darunter versteht man die Messung von Plasmakonzentrationen und eine Abschätzung der Pharmakokinetik des einzelnen Patienten. Mit dieser Information kann der Onkologe dann eine entsprechende maßgeschneiderte Dosisanpassung vornehmen. So bekommt dann jeder Patient genau die Dosis, bei der eine maximale Wirksamkeit bei minimalen Nebenwirkungen zu erwarten ist. Das wird in Deutschland leider noch relativ selten angewendet. Hier gibt es aber eine sehr interessante neue Entwicklung: Schon lange ist bekannt, dass Patienten, die Fluorouracil (5-FU) erhalten, sehr unterschiedliche Plasmakonzentrationen bei gleicher Dosis aufweisen. Dementsprechend unterschiedlich fällt die Wirkung von 5-FU aus. Während eine Überdosierung dem Behandler meist durch Nebenwirkungen auffällt, bleibt eine Unterdosierung in der Regel unbemerkt. Wir haben dazu vor einigen Jahren eine multizentrische Studie durchgeführt und waren selbst überrascht, dass $64 \%$ der Patienten unterdosiert waren [3]. Deshalb ist es sinnvoll, die Plasmakonzentrationen von 5-FU zu messen und über Modelle die optimale Dosis zu berechnen. Darüber hinaus gibt es verschiedene Genotypen des 5-FU-metabolisierenden Enzyms Dihydropyrimidin-Dehydrogenase (DPD). Dieser Polymorphismus ist dafür verantwortlich, dass manche Patienten 5-FU schnell verstoffwechseln, andere langsamer oder gar nicht. Die Deutsche Gesellschaft für Hämatologie und Onkologie hat 2020 ein Positionspapier herausgebracht, in dem eine DPD-Genotypisierung vor Beginn einer FU-haltigen Therapie empfohlen wird [4]. In bestimmten Fällen wird zusätzlich ein TDM empfohlen, um zu überprüfen, ob die Plasmakonzentration im richtigen Bereich liegt.

\section{I) Die DGHO empfiehlt eine DPD-Genotypisie- rung vor Beginn einer 5-FU-Therapie und in bestimmten Fällen ein Therapeutisches Drug Monitoring}

best practice onkologie: Wäre ein solches TDM nicht gerade bei oraler Tumortherapie wichtig, wenn die Patienten die Medikamente zu Hause einnehmen, ohne dass sie von Fachpersonal beobachtet werden?

U. Jaehde: Absolut richtig, im Rahmen der Central European Society for Anticancer Drug Research (CESAR) haben wir

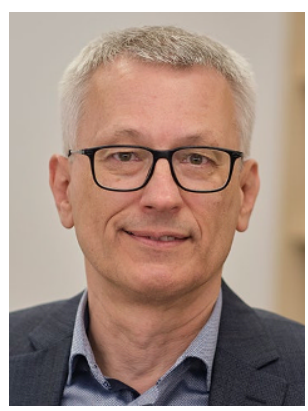

\section{Zur Person:}

Prof. Dr. Ulrich Jaehde studierte Pharmazie an der Freien Universität Berlin. Nach seiner Promotion forschte er zwei Jahre in der Abteilung für Pharmakologie der Universität Leiden, Niederlande. Anschließend kam er als wissenschaftlicher Mitarbeiter und wissenschaftlicher Assistent zurück nach Berlin und etablierte dort eine Arbeitsgruppe, die sich mit klinischpharmazeutischen Fragestellungen in der Onkologie beschäftigte. 1999 folgte er dem Ruf an die Rheinische Friedrich-Wilhelms-Universität Bonn und übernahm dort die erste Professur für Klinische Pharmazie in Deutschland. Vor 17 Jahren baute er in Bonn den neu gegründeten Bereich für Klinische Pharmazie auf. Parallel dazu engagierte er sich 1998-2002 als Vorsitzender der Arbeitsgemeinschaft Klinische Pharmazie in der Deutschen Pharmazeutischen Gesellschaft (DPhG). Seit 2007 ist er Mitglied im Wissenschaftlichen Beirat der Bundesapothekerkammer (BAK). Weiterhin war er von 2009 bis 2013 Präsident der Central European Society for Anticancer Drug Research (CESAR). 2009 wurde er sowohl in die Arzneimittelkommission der Deutschen Ärzteschaft (AkdÄ) als auch in die Arzneimittelkommission der Deutschen Apotheker (AMK) berufen. Seit 2013 ist er Mitglied der Koordinierungsgruppe für den Aktionsplan zur Verbesserung der Arzneimitteltherapiesicherheit (AMTS) des Bundesministeriums für Gesundheit. zusammen mit der Freien Universität Berlin und weiteren Universitäten ein Netzwerk zum TDM bei oraler Tumortherapie aufgebaut, das so genannte ON-TARGETProjekt. Das ist ein Projekt zum Mitmachen (s. Kasten). Beteiligen können sich Ärzte aus Kliniken und Praxen, indem sie Blutproben von Patienten unter oraler Tumortherapie an ein kooperierendes Labor einschicken. Dort wird die Konzentration der Substanz im Blutplasma analysiert, und die Werte werden über pharmakometrische Modelle ausgewertet. Der Arzt bekommt anschließend eine Rückmeldung, in welchem Konzentrationsbereich die Blutwerte des Patienten liegen. Auf diese Weise kann der Arzt nicht nur frühzeitig das Risiko für unerwünschte Wirkungen abschätzen, sondern bei seinen Patienten auch Arzneimittelwechselwirkungen oder mangelnde Therapietreue erkennen. Darauf basierend kann er den weiteren Therapieverlauf planen. Darüber hinaus untersuchen wir diese Real-World-Daten wissenschaftlich, um aufzeigen zu können, wie oft bestimmte Substanzen bei Patienten über- oder unterdosiert sind. Jede neu erhobene Konzentration verbessert die Vorhersagekraft unserer Modelle, das ist ein lernendes System. Wir fangen jetzt gerade mit den Substanzen Axitinib und Cabozantinib an, die für die Behandlung des Nierenzellkarzinoms eingesetzt werden. Unser Ziel ist es, in diesem Netzwerk nach und nach alle wichtigen oralen Tumortherapeutika abdecken zu können.

best practice onkologie: Ihre Arbeitsgruppe erstellt auch Betreuungsmodelle, die auf einem strukturierten Medikationsmanagement aufbauen. Wie lässt sich damit die Arzneimitteltherapiesicherheit bei Krebspatienten verbessern?

U. Jaehde: Wir möchten durch gezielte Maßnahmen den Medikationsprozess von der Verordnung bis hin zur Applikation am Patienten sicherer machen. Wir wissen inzwischen aus vielen Studien, dass im Rahmen des Medikationsprozesses viele Fehler passieren können, die man nachweislich am besten vermeiden kann, wenn die beteiligten Berufsgruppen, also unter anderem Ärzte, Apotheker und Pflegekräfte, möglichst unter Einbeziehung der Patienten optimal zusammenarbeiten. Das klingt einfach, aber in der Praxis wird es häufig noch nicht so umgesetzt. Unsere Modelle zielen darauf ab, die Aufgaben mittels Algorithmen ganz 


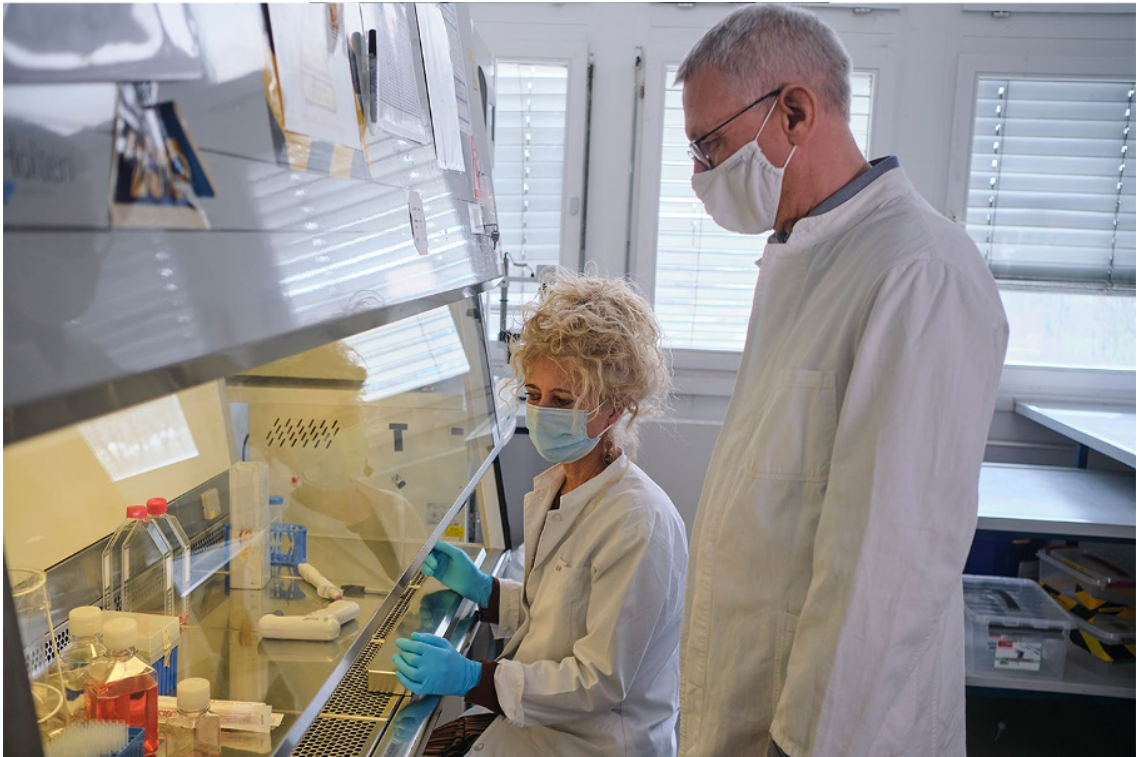

Durch regelmäßige Messung der Plasmakonzentrationen können Medikamente noch treffsicherer dosiert werden

klar einzelnen Berufsgruppen oder dem Patienten zuzuordnen, um Fehler und vor allem Nebenwirkungen zu vermeiden. Zum einen standardisieren die Modelle die Abläufe, zum anderen ermöglichen sie ein individualisiertes Vorgehen, indem bestimmte Maßnahmen erst ergriffen werden, wenn vorher definierte Kriterien vorliegen. Auch versuchen wir, diese Modelle mehr und mehr digital umzusetzen. Wahrscheinlich wird es die Digitalisierung sein, die dem Medikationsmanagement zum Durchbruch verhilft. Man kann die digitalisierten Modelle auch an Arzneimittelinformationssysteme koppeln, die es bereits in vielen Kliniken gibt. Darin steckt aus meiner Sicht großes Potenzial - auch für die Onkologie.

best practice onkologie: Apotheker können Krebspatienten ebenfalls zu Nebenund Wechselwirkungen von Medikamenten beraten. Findet sich hierfür in jeder Apotheke die Expertise?

U. Jaehde: Zunächst mal kann jede Apotheke Ärzte und Patienten zu Neben- und Wechselwirkungen beraten und hat auch das Know-how und das entsprechende Equipment dazu. Aber nicht alle Apotheken sind im Umgang mit Krebspatienten oder medikamentöser Tumortherapie gleichermaßen erfahren. Deshalb gibt es seit vielen Jahren eine Zusatzqualifikation für Fachapotheker. Die auf diesem Gebiet qualifizierten Apotheker erhalten damit die Zusatzbezeichnung „Onkolo- gische Pharmazie“ und sind sehr fit im onkologischen Bereich. Laut Bundesapothekerkammer haben diese Weiterbildung bis Ende 2019263 Apotheker abgeschlossen [5].

\section{I) Wahrscheinlich wird es die Digitalisierung sein, die dem Medikations- management zum Durchbruch verhilft}

best practice onkologie: Seit 2014 gibt es ein Grundsatzpapier der Bundesapothekerkammer zur Durchführung von Medikationsanalysen in Apotheken [6]. Wird dies in der Praxis auch umgesetzt?

U. Jaehde: Die Medikationsanalyse ist die strukturierte Überprüfung der Medikation eines Patienten. Dabei schaut der Apotheker, ob es arzneimittelbezogene Probleme wie ungeeignete Dosierungen oder Arzneimittelwechselwirkungen gibt. Seit Publikation dieses Grundsatzpapiers gibt es zahlreiche Modellprojekte - zum Beispiel ARMIN in Sachsen und Thüringen. In den Modellprojekten läuft die Medikationsanalyse seit vielen Jahren mit gutem Erfolg, aber wir sind in Deutschland leider noch weit entfernt von einer flächendeckenden Umsetzung. Der wich- tigste Grund hierfür ist sicherlich, dass die Krankenkassen diese Leistung noch nicht honorieren. Das könnte sich jetzt aber mit der Umsetzung des Ende 2020 verabschiedeten Vor-Ort-Apothekenstärkungsgesetzes ändern. Darin ist auch die Einführung pharmazeutischer Dienstleistungen festgeschrieben, die von den Krankenkassen honoriert werden. Die Bundesapothekerkammer wünscht sich unter anderem die Medikationsanalyse bei Patienten mit Polymedikation für diesen Dienstleistungskatalog. Darüber verhandelt der Deutsche Apothekerverband aber noch mit dem Spitzenverband der Krankenkassen.

\section{》) Mittels Medikations-} analyse und onkogeriatrischem Assessment kann man älteren Krebspatienten eine risikoadaptierte Betreuung anbieten

best practice onkologie: Eine Medikationsanalyse ist sicherlich auch für ältere Patienten sinnvoll, die ebenso im Fokus Ihrer Arbeit stehen. Welche Aspekte sind hier vordergründig?

U. Jaehde: Ältere Patienten unterliegen besonderen Risiken und sind letztendlich die Hauptkonsumenten von Medikamenten. Wenn ein Patient mindestens fünf Medikamente dauerhaft bekommt, sprechen wir von Polymedikation. Ungefähr die Hälfte der Krebspatienten, die 70 Jahre oder älter sind, gehen häufig schon mit einer Polymedikation in die Tumortherapie hinein. Gemeinsam mit oraler Tumortherapie und Supportivtherapien können auf diese Weise zu Beginn der Tumortherapie bis zu 15 Medikamente zusammenkommen, weshalb eine Medikationsanalyse gerade $\mathrm{zu}$ diesem Zeitpunkt sehr wichtig wäre. Wir testen derzeit im Johanniter-Krankenhaus Bonn ein interprofessionelles, risikoadaptiertes Medikationsmanagement für ältere Krebspatienten (IrMa). Darin fungiert eine Polymedikation sozusagen als Trigger für eine Medikationsanalyse. Parallel erfolgt ein onkogeriatrisches Assessment, um Patienten mit einem hohen Toxizitätsrisiko zu identifizieren. Anhand dieser beiden Kriterien bieten wir den älteren Krebspatienten eine 


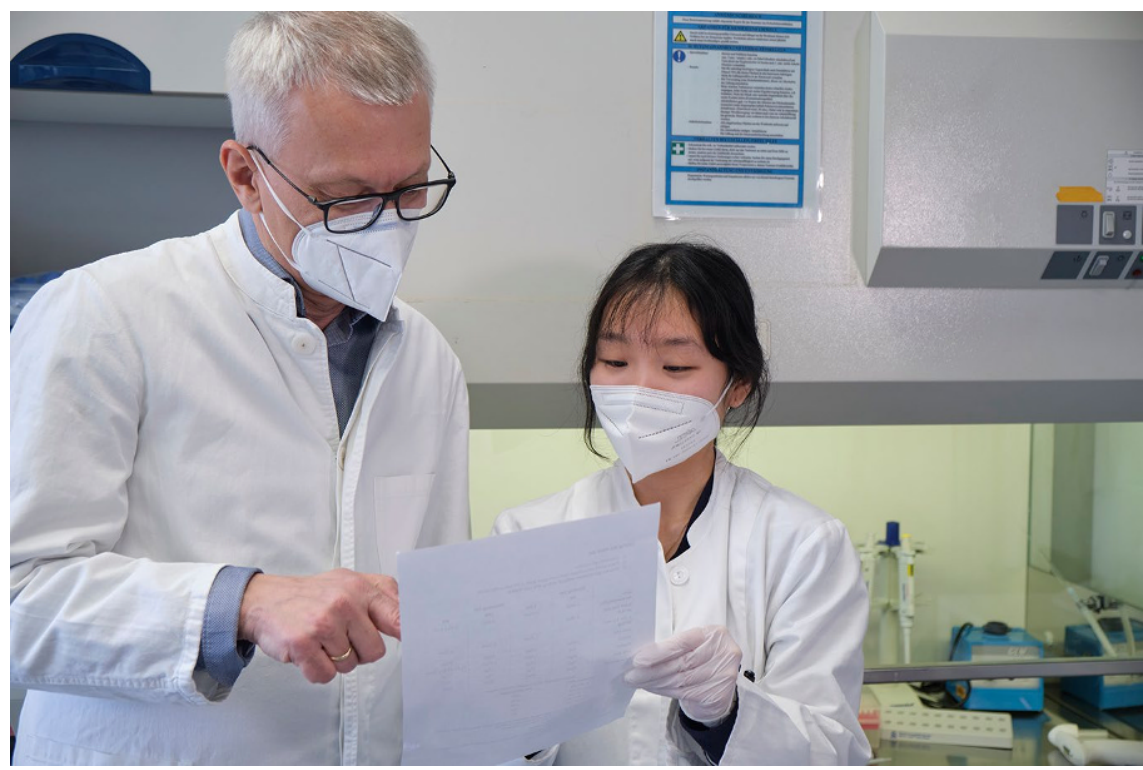

Überraschende Messergebnisse erfordern oft auch eine kurze Einschätzung zwischendurch

maßgeschneiderte Betreuung während der Tumortherapie an, mit denen therapieassoziierte Risiken, also vor allem Nebenwirkungen, reduziert werden können.

best practice onkologie: Sie sind Mitglied der Koordinierungsgruppe Aktionsplan zur Verbesserung der Arzneimitteltherapiesicherheit (AMTS) in Deutschland. Anfang Februar 2021 hat das Bundesministerium für Gesundheit (BMG) einen neuen Aktionsplan veröffentlicht [7]. Welche darin beschlossenen Maßnahmen sind entscheidend?

U. Jaehde: Diese Aktionspläne gibt es seit 2008, das ist jetzt der fünfte, der von 2021 bis 2024 gilt. Beschlossen wurden 42 ganz konkrete Maßnahmen. Ein wichtiger Schwerpunkt ist die Stärkung der Gesundheitskompetenz und der Patientenorientierung im Gesundheitswesen und dadurch eine bessere Einbindung der Patienten: also der Patient als Experte für seine Medikation, um die AMTS zu erhöhen. Der zweite entscheidende Schwerpunkt ist die Förderung der interprofessionellen Zusammenarbeit. In diesem Punkt kommen wir mit jedem Aktionsplan einen Schritt weiter, trotzdem sehen wir immer noch sehr viel Handlungsbedarf bezüglich einer klaren Rollenverteilung bei Medikationsanalyse und Medikationsmanagement. Es geht im Grunde darum, die Kompetenzen zu bündeln und gemeinsam das Beste für den Patienten herauszuholen. Ein weiterer Schwer- punkt ist es, digitale Möglichkeiten gezielt einzusetzen, damit AMTS nicht nur in Modellprojekten, sondern auch überall vor Ort ankommt. Auch die Onkologie spielt im neuen Aktionsplan eine Rolle. Das BMG wird ein Modellprojekt zur Implementierung und Evaluierung einer Interventionsstrategie zur Verbesserung der AMTS bei oraler Tumortherapie ausschreiben.

\section{》IIm Grunde geht es darum, die Kompetenzen zu bündeln und gemeinsam das Beste für den Patienten heraus- zuholen}

best practice onkologie: Was ist noch nötig, damit die Maßnahmen Ihrer Modellprojekte an der Basis ankommen?

U. Jaehde: Für ein gutes Medikationsmanagement und eine verbesserte AMTS braucht es auch die richtigen Anreize und vielleicht auch eine konkrete Pflicht, z. B. regelmäßig die Medikation zu überprüfen. Vielleicht bräuchte man eine Art Medikations- oder AMTS-Beauftragten vor Ort, der dafür verantwortlich ist, dass in der jeweiligen Institution alles getan wird, um die AMTS hochzuhalten. Und natür- lich ist es wichtig, dass die Professionen zusammenarbeiten: Man hat den Arzneimittelexperten, also den Apotheker, in der Nähe, und diese Kompetenz sollte man auch nutzen.

\section{》) Die Apotheker wären mit einer intensiveren klinischeren Ausbildung noch besser vorbereitet, Patienten und Ärzte in der Therapie zu unterstützen}

best practice onkologie: Wie sollte die Zusammenarbeit von Apotheker und Onkologen aussehen?

U. Jaehde: In jedem Fall sollte sie so eng wie möglich sein. In einer ganzen Reihe von Krankenhäusern arbeiten Apotheker mit im therapeutischen Team, gehen mit auf die Visite und sind dort zuständig für die richtige Dosierung und Anwendung von Medikamenten. Sie gestalten also gemeinsam mit dem Onkologen und den anderen Berufsgruppen die Therapie eines Patienten. Im ambulanten Bereich ist das noch selten der Fall, aber es gibt auch hier gute Beispiele für eine enge $\mathrm{Zu}$ sammenarbeit zwischen niedergelassenen Onkologen und öffentlichen Apotheken. Allerdings steht auch hier die Flächendeckung noch aus. Möglicherweise wird sich über die neuen gesetzlichen Rahmenbedingungen etwas ändern. Es gibt viele angehende Apotheker, die genau das machen wollen. Sie wollen zusammen mit dem Arzt die Therapie begleiten und damit wirksamer und sicherer machen. Man muss nur die erforderlichen Strukturen dafür schaffen.

best practice onkologie: Seit 2004 sind Sie Leiter des Bereichs Klinische Pharmazie an der Universität Bonn. Werden die angehenden Pharmazeuten ausreichend auf die interprofessionelle Arbeit vorbereitet?

U. Jaehde: Das Pharmaziestudium ist überwiegend naturwissenschaftlich ausgerichtet. Mit der letzten Änderung im Jahr 2001 wurde die Approbationsordnung für Apotheker geändert, und es wurde das Fach Klinische Pharmazie eingeführt. Seinerzeit war das hier an der Universität Bonn die erste Professur für 


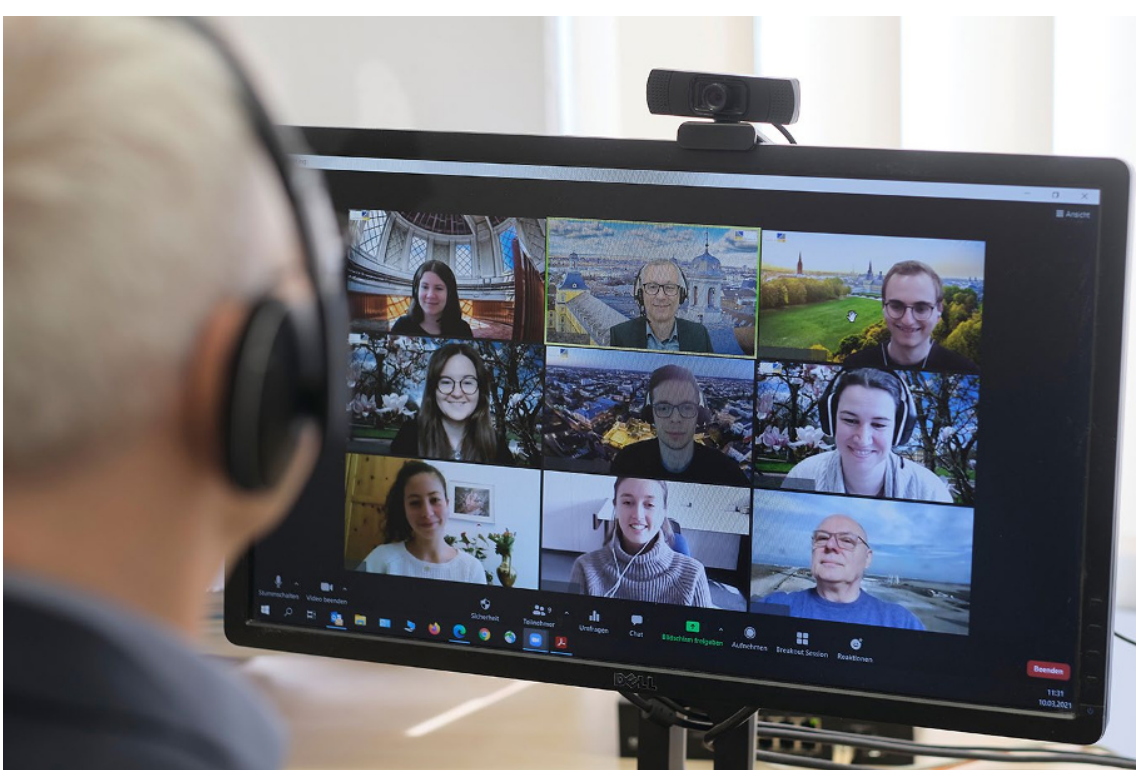

In Zeiten von COVID-19 werden auch sämtliche Teambesprechungen im Pharmazeutischen Institut digital abgehalten

\section{Mitmachprojekt ON-TARGET}

Das Projekt "ON-TARGET“ im Rahmen der "Central European Society for Anticancer Drug Research" (CESAR) bietet ein Therapeutisches Drug Monitoring bei Patienten mit oraler Tumortherapie an. Ärzte aus Klinik und Praxis können Blutproben an kooperierende Labore schicken und erhalten dann eine Rückmeldung zu den im Blut ihres Patienten gemessenen Arzneistoffkonzentrationen. Damit kann frühzeitig die Wahrscheinlichkeit für schwere unerwünschte Arzneimittelwirkungen abgeschätzt und die Therapie entsprechend angepasst werden. Dafür werden Arzneistoffkonzentrations-Schwellenwerte genutzt, die in klinischen Studien mit einem erhöhten Auftreten von unerwünschten Arzneimittelwirkungen in Zusammenhang gebracht wurden (www.fu-berlin.de/ontarget). Für weitere Informationen wenden Sie sich bitte an Prof. Jaehde unter der E-Mail-Adresse: u.jaehde@uni-bonn.de.

das Fach in Deutschland, also bekam ich die Gelegenheit, die Lehre in diesem Fach von Null an aufzubauen. Seitdem sind in der Klinischen Pharmazie viele Inhalte dazugekommen. Im letzten Semester beschäftigen sich die Studierenden überwiegend mit der Arzneimitteltherapie einzelner Patienten. Das heißt also, dass die Apotheker, die ab 2001 studiert haben, ein gutes Fundament an Kenntnissen zur Arzneimitteltherapie haben. Das lässt sich aber noch ausbauen. Derzeit wird sehr viel über eine Novellierung der Approbationsordnung für Apotheker diskutiert. Es wird immer wieder gefordert, dass gerade die Klinische Pharmazie und die Pharmakologie gestärkt werden sollen. Die Apotheker wären mit einer intensiveren klinischeren Ausbildung natürlich noch besser vorbereitet, Patienten und Ärzte in der Therapie zu unterstützen.

best practice onkologie: Herr Professor Jaehde, vielen Dank für das interessante Gespräch.

Das Interview führte: Sabrina Kempe, Dresden

Fotos: Heiko Specht

\footnotetext{
Literatur

1. Hagelstein Vet al (2016) Validation of the German patient-reported outcomes of the common terminology criteria for adverse events (PRO-CTCAE). Ann Oncol 27(12):2294-2299

2. Absolom Ket al (2021) Phase III randomized controlled trial of eRAPID: ehealth intervention during chemotherapy. J Clin Oncol 39(7):734-747

3. Wilhelm M et al (2016) Prospective, multicenter study of 5-fluorouracil therapeutic drug monitoring in metastatic colorectal cancer treated in routine clinical practice. Clin Colorectal Cancer 15(4):381-388

4. Wörmann B et al (2020) Dihydropyrimidine dehydrogenase testing prior to treatment with 5-fluorouracil, capecitabine, and tegafur: a consensus paper. Oncol Res Treat 43(11):628-636
}

5. ABDA - Bundesvereinigung Deutscher Apothekerverbände (Hrsg) (2020) Die Apotheke - Zahlen, Daten, Fakten 2020. Berlin

6. $A B D A$ - Bundesvereinigung Deutscher Apothekerverbände (Hrsg) (2014) Grundsatzpapier zur Medikationsanalyse und zum Medikationsmanagement. Berlin

7. Bundesministerium für Gesundheit (2021) Aktionsplan 2021-2024 des Bundesministeriums für Gesundheit zur Verbesserung der Arzneimitteltherapiesicherheit in Deutschland, Bonn. https://www. bundesgesundheitsministerium.de/fileadmin/Dateien/5_Publikationen/Gesundheit/Berichte/Aktionsplan_2021-2024_BMG_AMTS.pdf.Zugegriffen: 21. Apr. 2021 